\title{
Acoustoelectric Current in Graphene Nanoribbon due to Landau Damping
}

K. A. Dompreh

University of Cape Coast

K.W.Adu ( $\nabla$ cxa269@psu.edu )

Pennsylvania State university-Altoona College

D. Sakyi-Arthur

University of Cape Coast

N. G. Mensah

University of Cape Coast

S. Y. Mensah

University of Cape Coast

A Twum

University of Cape Coast

M. Amekpewu

C. K. Tedam University of Applied Sciences and Technology

\section{Research Article}

Keywords: Acoustoelectric current, Landau Damping, Cerenkov Emission, Graphene Nanoribbon

Posted Date: May 14th, 2021

DOI: https://doi.org/10.21203/rs.3.rs-500367/v1

License: (c) (1) This work is licensed under a Creative Commons Attribution 4.0 International License.

Read Full License 


\title{
Acoustoelectric Current in Graphene Nanoribbon due to Landau Damping
}

\author{
K. A. Dompreh ${ }^{1}$, K. W. Adu ${ }^{2,3 *}$, D. Sakyi-Arthur ${ }^{1}$, N. G. Mensah ${ }^{4}$, S. Y. Mensah ${ }^{1}$, A. \\ Twum 1 , M. Amekpewu ${ }^{5}$ \\ ${ }^{1}$ Department of Physics, College of Agriculture and Natural Sciences, University of Cape Coast, Ghana. \\ ${ }^{2}$ Department of Physics, Pennsylvania State university-Altoona College, Altoona, Pennsylvania 16601, USA \\ ${ }^{3}$ Material Research Institute, Pennsylvania State University, University Park, Pennsylvania 16802, USA \\ ${ }^{4}$ Department of Mathematics, College of Agriculture and Natural Sciences, U.C.C, Cape Coast, Ghana \\ ${ }^{5}$ Department of Applied Physics, C. K. Tedam University of Applied Sciences and Technology, Navrongo, \\ Ghana.
}

\section{Abstract}

We perform self-consistent analysis of the Boltzmann transport equation for momentum and energy in the hypersound regime i.e., $q l>>1$ ( $q$ is the acoustic wavenumber and $l$ is the mean free path). Here, we investigate Landau damping of acoustic phonons $(L D O A P)$ in graphene nanoribbon that leads to acoustoelectric current generation. Under a non-quantized field with drift velocity, we observed an acoustic phonon energy quantization which depends on the energy gap, the width and the sub-index of the material. An effect similar to Cerenkov emission was observed where the electron absorbs the confined acoustic phonons energy, causing the generation of acoustoelectric current in Graphene Nanoribbon. A qualitative analysis of the absorption and versus phonon frequency is in agreement with experimental reports. We observed a shift in the peaks when the energy gap and the drift velocity were varied. Most importantly, a transparency window appears when making graphene nanoribbon a potential candidate as an acoustic wave filter with applications in phonon spectrometers and also as tunable gate-controlled quantum information device.

Key Words: Acoustoelectric current, Landau Damping, Cerenkov Emission, Graphene Nanoribbon

\footnotetext{
${ }^{*}$ Correspondence to [cxa269@psu.edu]
} 


\section{Introduction}

Landau Damping of Plasma Waves $(L D O P W)$ [1] is a phenomenon that involves the loss of energy from a collective motion of plasma waves to individual particles, where plasmons decay by exciting an electron below the Fermi level. The mechanism of $\angle D O P W$ is observed in various systems from plasma oscillations (Langmuir wave) to accelerators. Even though this phenomenon is well known to occur in plasmons, it can also occur during electron-phonon interactions. In semiconductors, Landau Damping of acoustic phonons ( $L D O A P$ ) has been studied using Raman spectroscopy [2] and electron energy loss spectroscopy [3].

In electron-phonon interactions, the transfer of momentum from phonons to the conducting charge carriers leads to the generation of $d . c$ current in the material, known as acoustoelectric effect ( $A E$ ) [4-6]. Theoretical and experimental studies of the generation of $A E$ in low dimensional structures such as graphene [7-10], quantum wells $(Q W)[11]$ and carbon nanotubes [12-14, 28-30] as well as superlattices $(S L)[4,15]$ have generated lots of interest recently due to the variety of applications that can be derived from these novel nanostructures. Much of the theoretical investigations are mostly pursued using microscopic theory of electron-phonon interactions employing semi-classical Boltzmann's transport equation in the hypersound regime [4,5]. One method mostly used in the generation of $A E$ is Cerenkov emission $(C E)$ [16, 17], that is where a non-quantizing electric field applied to a material leads to amplification or absorption of the acoustic phonons when $v_{D}>v_{s}$ and $v_{D}<v_{s}$, respectively as demonstrated in the generation of SASER [16]. Mensah et. al., proposed theoretically the amplification of acoustic phonons via $C E$ in semiconductor superlattice $(S S L)$, and was confirmed experimentally by Shinokita et. al. where they achieved $200 \%$ increase in amplification of acoustic phonons [18].

From the conservation of energy and momentum, the electrons colliding with the acoustic phonons under a drift field also interact with the generated sound waves. Therefore, considering the acoustic waves as phonons of frequency $\left(\omega_{q}\right)$, the conducting electrons can absorb the acoustic phonons energy. This leads to the damping of the 
acoustic phonons and subsequently the production of acoustoelectric current. As the frequency of the acoustic phonon increases, the acoustoelectric current increases until there is resonance beyond which the absorption decreases. The absorption of the phonon energy is determined by the energy balance of the system, similar to $L D O P W$. This phenomenon has been observed in a number of graphene based $A E$ experiments and used in the fabrication of sensing devices such as humidity sensors [18], photodetectors [19], and gas sensors [20]. To the best of our knowledge, there is no theoretical investigation of Landau Damping of Acoustic Phonon due to Cerenkov Emission ( $L D O C E$ ) in GNR up-to-date, even though some experimental evidence of the phenomenon exist [8]. Poole, et. al [8] reported a nonlinear $A E$ in GNR of width $500 \mathrm{~nm}$ when stimulated with a d.c current. They observed a resonance between the measured current maxima after which the current decreased. Liang et.al [21], Zheng, et. al [27] and Okuda, et. al. [22] reported similar behaviour, where the acoustic charge transportation is induced by surface acoustic wave $(S A W)$ propagation in the graphene. Morgado, et. al. [23] also, reported Negative Landau Damping ( $N L D)$ in bilayer graphene. This was obtained by applying a $d . c$ electric current induced by a static voltage across the graphene sheet. Patterning single layer graphene (SLG) into GNRS opens an energy gap $(\Delta)$ which varies with the width, where the confined acoustic phonon energy is quantized. In this work, we study the nonlinear $A E$ in $S L G$ and $G N R-500$ with degenerate energy dispersion in the hypersound regime. Herein, we examine theoretically the effect of temperature $(T)$, thermal gradient $(\nabla T)$ and $d . c$ fields to investigate the $L D O A P$ in a $S L G$ and $G N R-500$ and quantitatively compare the results to existing experimental data of acoustoelectric current generation in Chemical Vapor Deposition (CVD) grown monolayer graphene.

\section{Methods, Results and Discussion}

To gain insights into $L D O A P$ in $S L G$ and $500-G N R$, we analyse the effect of electronphonon interaction in a gate-controlled single sheet of graphene. An in-plane current is applied along the axis of the graphene sheet where the electronic transition rate induced by electron interaction with acoustic phonons gives the kinetic equation for the acoustic phonon population $N_{q}(t)[4,5,6,17]$, expressed as: 


$$
\begin{aligned}
& \frac{\partial N_{q}}{\partial t}=\frac{2 \pi}{\mathrm{h}} g_{s} g_{v} \sum_{k, k^{\prime}}\left|C_{q}\right|^{2} \delta_{k, k^{\prime}}\left\{\left|N_{q}(t)+1\right| f_{k}\left(1-f_{k^{\prime}}\right) \delta\left(\varepsilon_{k^{\prime}}-\varepsilon_{k}+\mathrm{h} \omega_{q}\right)-\right. \\
& \left.N_{q}(t) f_{k^{\prime}}\left(1-f_{k}\right) \delta\left(\varepsilon_{k^{\prime}}-\varepsilon_{k}-h \omega_{q}\right)\right\}
\end{aligned}
$$

here, the spin and valley degeneracies are $g_{s}=2$ and $g_{v}=2$ respectively, $C_{q}=\sqrt{|\Lambda|^{2} \mathrm{~h} q / 2 \rho \mathrm{h} \omega_{q}}$ with $\Lambda$ being the deformation potential, $\rho$ is the density of the graphene sheet, and $\tau$ is the relaxation constant. The factor $f_{k}\left(1-f_{k^{\prime}}\right)$ is probability that the initial state $k$ is occupied and the final electron state $k^{\prime}$ is empty. $f_{k}$ is the unperturbed Fermi-Dirac distribution function, the factor $N_{q} f_{k^{\prime}}\left(1-f_{k}\right)$ is that of the boson and fermion statistics and the $\varepsilon_{k, k^{\prime}}$ is the energy dispersion. The summation in Eqn. (1) is over $k, k^{\prime}$ and can be transformed into integrals as

$$
\sum_{k, k^{\prime}} \rightarrow \frac{A^{2}}{(2 \pi)^{4}} \int d^{2} k d^{2} k^{\prime}
$$

Where, $A$ is the area of the material. Considering $N_{q}(t)>>1$, yields $\frac{\delta N_{q}}{\delta t}=\Gamma_{q} N_{q}$, where, $\Gamma_{q}$ is the absorption coefficient, expressed as:

$$
\Gamma_{q}=\frac{A\left|C_{q}\right|^{2} \mathrm{~h} q}{2 \pi^{3} \mathrm{~h} v_{f} \rho v_{s}} \int_{0}^{\infty} k d k \int_{0}^{\infty} k^{\prime} d k^{\prime} \int_{0}^{2 \pi} d \varphi \int_{0}^{2 \pi}\left\{d \theta\left[f(k)-f\left(k^{\prime}\right)\right] * \delta\left(k-k^{\prime}-\frac{1}{\mathrm{~h} v_{f}}\left(\mathrm{~h} \omega_{q}+v_{D} \mathrm{~h} q\right)\right)\right\}
$$

Considering an $S L G$ with linear energy dispersion given as $\varepsilon(k)= \pm h v_{f}|k|$, we first analyse the effect of temperature change on the mobility of electrons in the SLG by switching off the applied voltage. This enables the study of the effect of carrier concentration under different temperatures. As a consequence of energy conservation in the electron-phonon scattering process $k^{\prime}=k-\frac{1}{\mathrm{~h} v_{f}}\left(\mathrm{~h} \omega_{q}\right)$, the electron velocity is obtained by differentiating the energy dispersion, i.e., $v_{i}=\frac{2 \mathrm{~h} \omega_{q}}{\mathrm{~h} v_{f}}$. Considering the condition that $k_{B} T=1$, the Fermi - Dirac distribution becomes $f(k)=\exp (-\beta(\varepsilon(k)))$ 
with $\beta=1 / k_{B} T$, where $k_{B}$ is the Boltzmann constant. The absorption coefficient relates to the $A E$ current via the Weinrich [25] relation as

$$
J_{a c}=-\frac{2 e \tau}{\mathrm{h} v_{f}} \Gamma_{q}
$$

where $\tau$ is the relaxation time. Multiplying Eqn. (3) by $v_{i}$ gives the acoustoelectric current $J_{a c}$, as

$$
J_{a c}=-\frac{A\left|C_{q}\right|^{2} e \tau \mathrm{h} \omega_{q}}{2 \pi^{3} \mathrm{~h} v_{f} \rho v_{s}} \int_{0}^{2 \pi} k d k\left(k-\frac{1}{\mathrm{~h} v_{f}}\left(\mathrm{~h} \omega_{q}\right)\right)\left[\exp \left(-\beta \mathrm{h} v_{f} k\right)-\exp \left(-\beta \mathrm{h} v_{f}\left(k-+\frac{1}{\mathrm{~h} v_{f}}\left(\mathrm{~h} \omega_{q}\right)\right]\right.\right.
$$

Integrating and simplifying in Eqn. (5) yields

$$
J_{a c}=J_{0}\left\{2-\beta \mathrm{h} \omega_{q}\right\}\left[1-\exp \left(-\beta \mathrm{h} \omega_{q}\right)\right]
$$

where $J_{0}=\frac{2 \tau A|\Lambda|^{2} k T h \omega_{q}}{(2 \pi)^{3} \beta^{3} h^{4} v_{f}^{4} \rho V_{s}}$
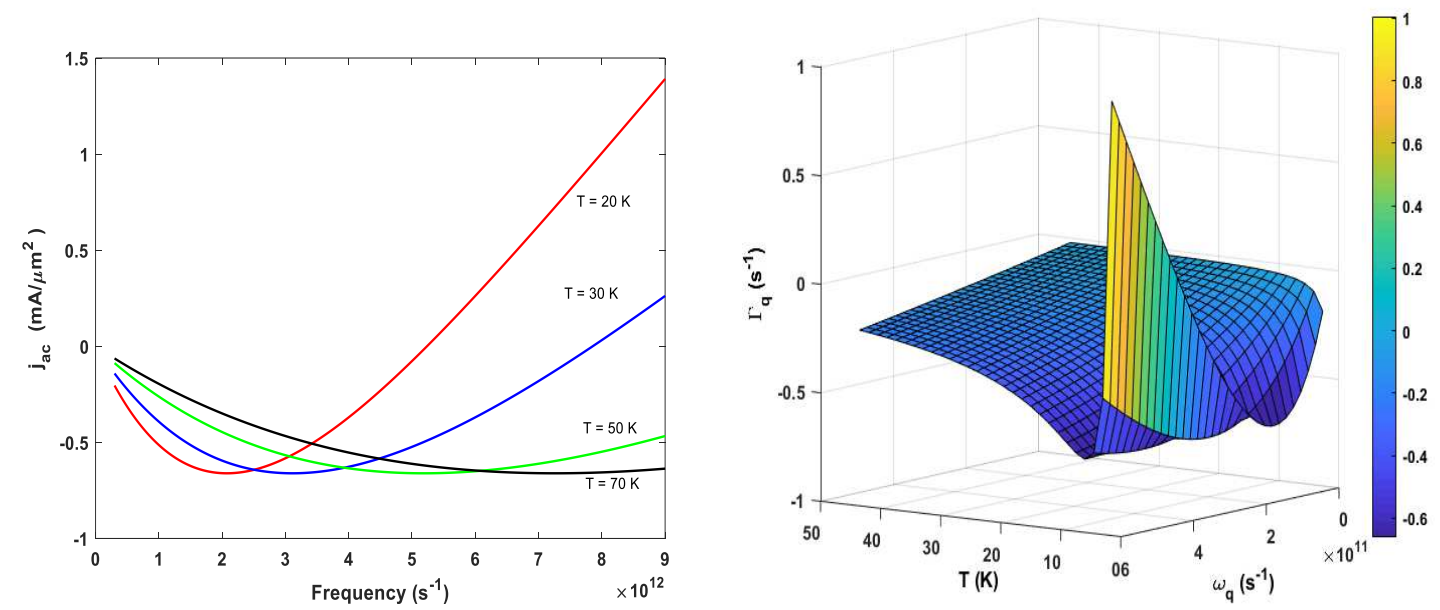

Figure 1: (a) Dependence of $J_{a c}$ on $\omega_{q}$ for various temperatures $(T)$, and (b) a 3D plot of $\Gamma_{q}$ versus $\omega_{q}$ and $T$.

From Eqn. (6), the $J_{a c}$ scales with temperature as $T^{4}$, which according to [24] indicates the contribution of an in-plane acoustic phonon. In Fig. 1a we show the dependence of $J_{a c}$ on frequency using the following parameters, $V_{F} \approx 10^{8} \mathrm{~ms}^{-1}, \tau=5 * 10^{-10} \mathrm{~s} \quad \Lambda=9 \mathrm{eV}$, $V_{s}=2.1 \times 10^{3} \mathrm{cms}^{-1}$ and $\mathrm{q}=10^{5} \mathrm{~cm}^{-1}$ for different temperatures, $\mathrm{T}=20,30,40$ and $70 \mathrm{~K}$. The plot 
shows a non-linear $A E$ current $J_{a c}$ which decreases with an increase in temperature. The acoustoelectric current does not exhibit a simple linear dependence on $\omega_{q}$, and temperature. At $\mathrm{T}=20 \mathrm{~K}$, the current initially decreased to a minimum at $2 \mathrm{THz}$, and then increases at higher frequencies. The same trend is observed when the temperature is increased, but $J_{a c}$ decreases. That is, increasing the temperature leads to a decrease in the current. This indicates the transport of holes in the material, and as the temperature increases, the lattice vibration also increases, limiting the flow of the acoustoelectric current. Using the relation $I=\mathrm{h} \omega_{q}$, the intensity of the acoustic phonons is directly proportional to its frequency $\left(\omega_{q}\right)$ thus, Fig. 1a is qualitatively in agreement with the experimental work by Bandhu and Nash [9]. The intensity initially decreases to a minimum and then increases as the frequency increases. To further illustration, the simultaneous dependence of the $\Gamma_{q}$ on frequency $\left(\omega_{q}\right)$ and temperature $(T)$ is shown as a $3 \mathrm{D}$ plot in Fig. 1b. For the $\Gamma_{q}$ on $T$, the graph decreased to a minimum and then increased to a point and remained constant at higher temperatures whilst that of the $\Gamma_{q}$ on $\omega_{q}$ conformed to that of Fig.1a.

By switching on the drift field, $k^{\prime}=k-\frac{1}{\mathrm{~h} v_{f}}\left(\mathrm{~h} \omega_{q}+v_{D} \cdot \mathrm{h} q\right)$, Eqn. (3) becomes

$\Gamma_{q}=\Gamma_{0}\left\{2-\beta \mathrm{h} \omega_{q}\left(1-\frac{v_{D}}{v_{s}}\right)\right\}\left[1-\exp \left(-\beta \mathrm{h} \omega_{q}\left(1-\frac{v_{D}}{v_{s}}\right)\right)\right]$

where $\Gamma_{0}=J_{0}$. The Eqn. (7) is analysed numerically for a normalized $\Gamma_{q}$ dependence on $v_{D} / v_{s}$ and $\omega_{q}$. Shown in Fig.2a is the dependence of $\Gamma_{q}$ on $\omega_{q}$ that depicts a linear relationship. 

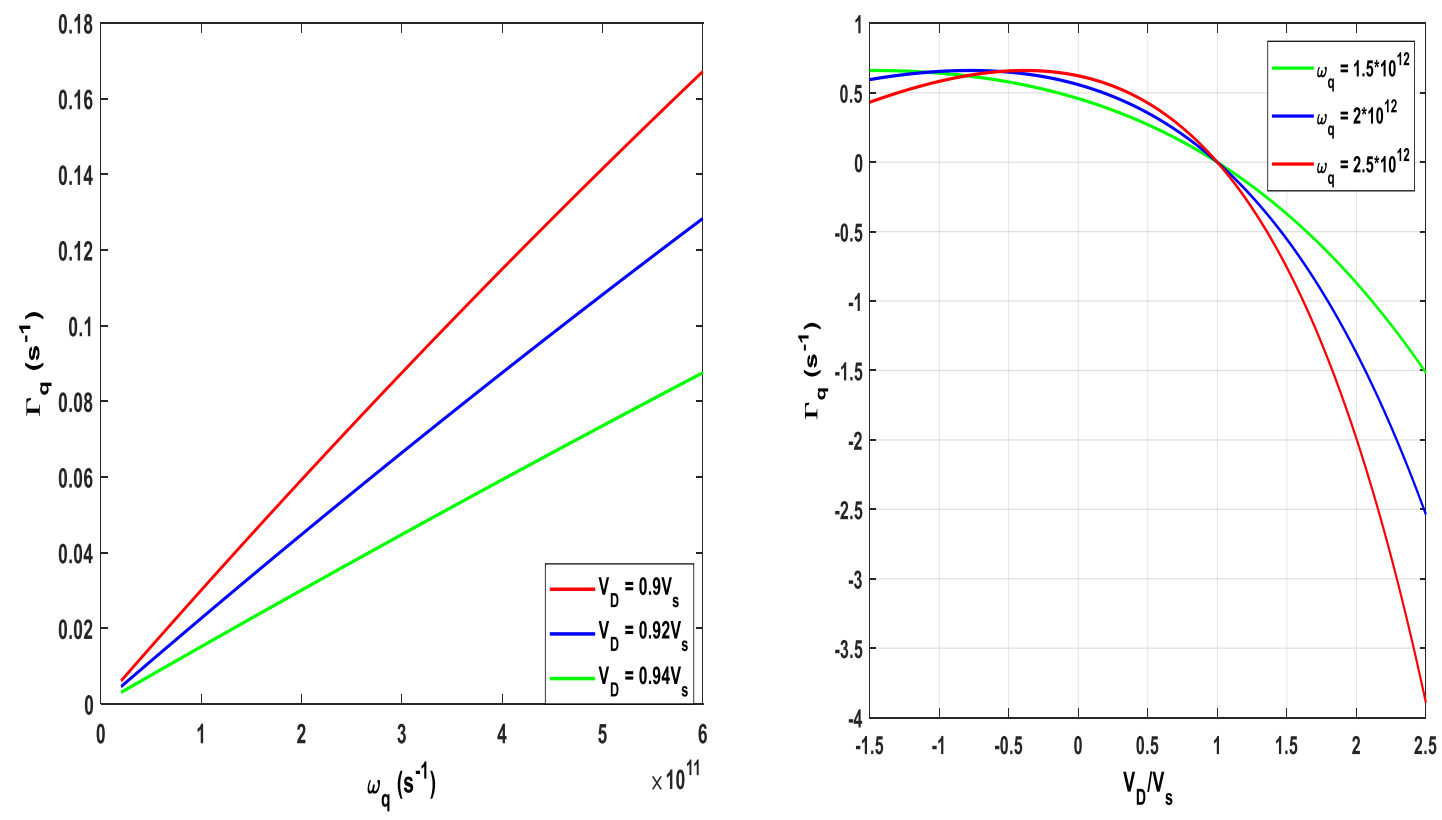

Fig2 (a) The dependence of $\Gamma_{q}$ on $\omega_{q}$ for $v_{\mathrm{D}}=0.9,0.92,0.94$, and (b) the dependence of $\Gamma_{q}$ on $v_{D} / v_{s}$ at varying $\omega_{q}=1.2 \mathrm{THz}, 2 \mathrm{THz}, 2.2 \mathrm{THz}$

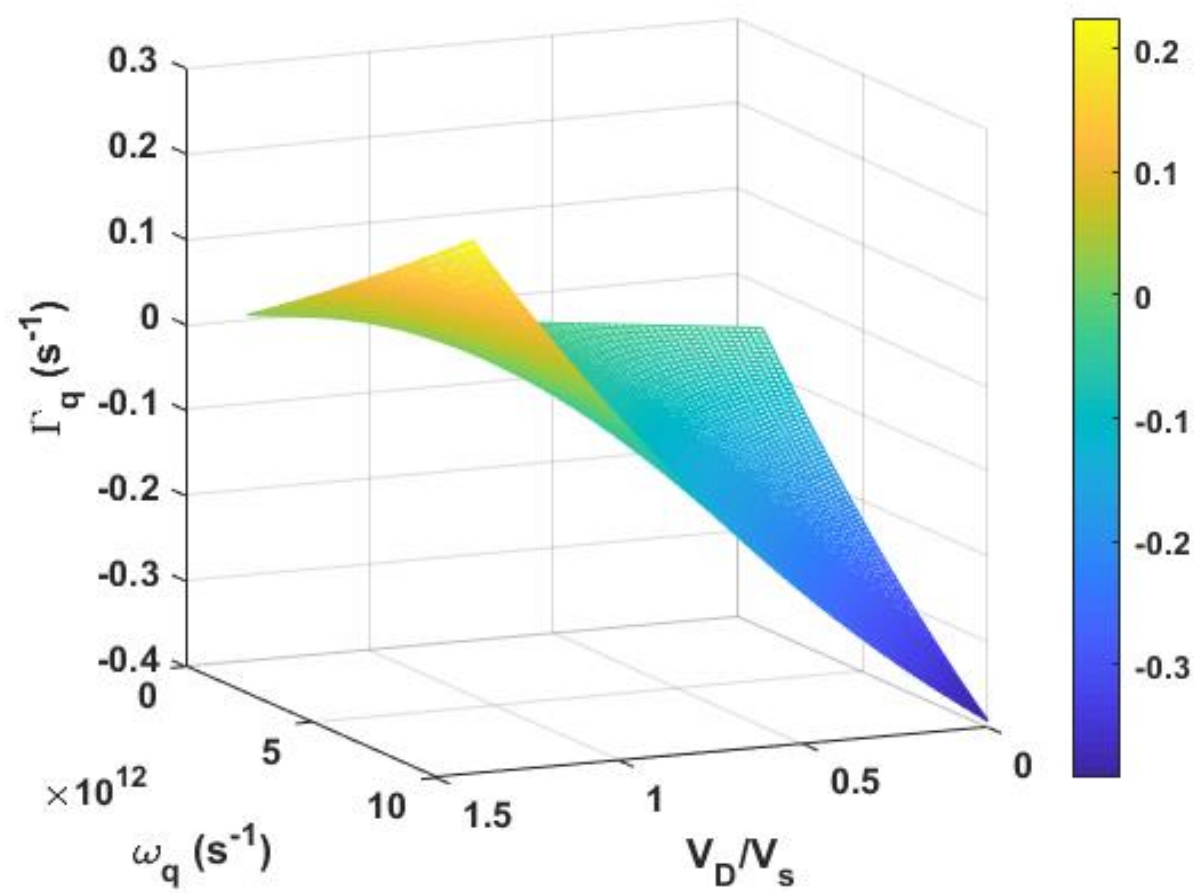

Fig. 2c: A 3D plot of the dependence of $\Gamma_{q}$ on $v_{D} / v_{s}$ and $\omega_{q}$ 
Fig.2a shows the graph of the dependence of $\Gamma_{q}$ on $\omega_{q}$ for various values of $v_{\mathrm{D}}=0.9,0.92$ and 0.94. The absoption showed linear relationship with the frequency but decreases when the $v_{D}$ was increased.

Using the Weinriech relation as in Eqn (4), .Fig. 2a qualitatively agrees with the experimental report in ref. [9] where the acoustoelectric current varied linearly with the frequency. When a non-quantizing electric field is applied along the axis of $S L G$, for $v_{D}>v_{s}$, an amplification of acoustic phonons is obtained, and the reverse, $v_{D}<v_{s}$ leads to the absorption of acoustic phonons. This phenomenon is similar to the Cerenkov's emission of phonons. Fig. $2 \mathrm{~b}$ shows the dependence of $\Gamma_{q}$ on $v_{D} / v_{s}$ for different values of $\omega_{q}$. An absorption occurs when $v_{D} / v_{s}<1$ and amplification happens when $v_{D} / v_{s}>1$, consistent with the work of Nunes and Fonseca [32]. The dependence of $\Gamma_{q}$ on $v_{D} / v_{s}$ and $\omega_{q}$ is shown in Fig. 2c. Numerically, setting the $v_{D}=1.1 v_{s}$, the maximum amplification is obtained at $\Gamma_{q}=-0.16$ at $\omega_{q}=2 T H z$. It is interesting to note that, our results is in good agreed with the work in ref. [27] where acousticphonon frequencies above $10 \mathrm{THz}$ were attained. The field $E$ in the material can be calculated by using $E=v_{D} / \mu$, where $\mu=2.0 \times 10^{4} \mathrm{~cm}^{2} / v_{s}$ is the electron mobility in graphene. Using $v_{s}=2.1 \times 10^{5} \mathrm{~cm} / \mathrm{s}$ gives $E=11.5 \mathrm{~V} / \mathrm{cm}$. For the source-to-drain voltage, $V_{s d}=v_{D} \mathrm{~L} / \mu,(L$ is the length from the source to the drain electrode in the graphene), the in-plane current $I=$ $e n v_{D} L$ ( $n$ is the electron density) can be calculated. Patterning graphene into GNR (one dimensional material) opens a band-gap with the energy dispersion given as

$$
\varepsilon(k)=\frac{\Delta}{2} \sqrt{1+\frac{\mathrm{h}^{2} k^{2}}{(\mathrm{~h} B)^{2}}}
$$

where the $\Delta$ is the energy gap and $B$ is the quantized wave vector. By considering that the acoustic phonon and the electric field are directed along the GNR axis, $k^{\prime}=(k+h q) \cos \theta$, where $\theta$ is the scattering angle. When a field is applied to the GNR, the energy level become degenerate and at low temperatures when $\varepsilon(p) \gg h \omega_{q}$, the $(h q)^{2} \rightarrow 0$ and Eqn. (1) becomes (see Supplementary information) 


$$
\begin{aligned}
& \Gamma_{q}=-\frac{\pi C_{q}^{2} \Delta \mathrm{h} q}{2 \mathrm{~h}^{2} B^{2}}\left\{\left[1+\frac{1}{4(\mathrm{~h} B)^{2}}\left(-\mathrm{h} q \cos (\theta)+\sqrt{4\left(2(\mathrm{~h} B)^{2}-\frac{2 \mathrm{~h}^{4} \omega_{q}^{2} B^{2}}{\Delta^{2}}\left(1-\frac{v_{D}}{v_{s}}\right)^{2}\right.}\right)\right)^{2}\right]^{-\frac{1}{2}} * \\
& \frac{1}{2}\left(-\mathrm{h} q \cos (\theta)+\sqrt{4\left(2(\mathrm{~h} B)^{2}-\frac{2 \mathrm{~h}^{4} \omega_{q}^{2} B^{2}}{\Delta^{2}}\left(1-\frac{v_{D}}{v_{s}}\right)^{2}\right)}\right)- \\
& \left.\left[1+\frac{1}{4(\mathrm{~h} B)^{2}}\left(-\mathrm{h} q \cos (\theta)-\sqrt{4\left(2(\mathrm{~h} B)^{2}-\frac{2 \mathrm{~h}^{4} \omega_{q}^{2} B^{2}}{\varepsilon_{g}^{2}}\left(1-\frac{v_{D}}{v_{s}}\right)^{2}\right.}\right)\right]^{2}\right]^{-\frac{1}{2}} * \\
& \left.\frac{1}{2}\left(-\mathrm{h} q \cos (\theta)-\sqrt{4\left(2(\mathrm{~h} B)^{2}-\frac{2 \mathrm{~h}^{4} \omega_{q}^{2} B^{2}}{\Delta^{2}}\left(1-\frac{v_{D}}{v_{s}}\right)^{2}\right)}\right)\right\} * \\
& \left(\sqrt{4\left(2(\mathbf{h} B)^{2}-\frac{2 \mathbf{h}^{4} \omega_{q}^{2} B^{2}}{\Delta^{2}}\left(1-\frac{v_{D}}{v_{s}}\right)^{2}\right)}\right)^{-1}
\end{aligned}
$$

In Eqn. (9), $(\mathrm{h} B)^{2}$ is the quantized acoustic phonon energy, $B=\frac{2 \pi}{a_{c-c} \sqrt{3}}\left(\frac{P i}{N+1}-\frac{2}{3}\right), N$ is the width of the graphene, $a_{c-c}$ is the lattice constant and $P i$ is the sub-band index. The absorption reveals the characteristic feature of the acoustic phonon spectrum in the materials that occurs in the Terahertz frequency range. Taking $N \approx 500 \mathrm{~nm}, \Delta=0.1,0.2,0.3,0.4 \mathrm{eV}, v_{D}<v_{s}$ and the parameters used in Fig.1, the plot of $\Gamma_{q}$ versus $\omega_{q}$ as in Eqn. (9) is shown in Fig. 3. The plot shows two absorption peaks with the gap between the peaks. The absorption increases as the energy gap increases, similar to the experimental report in ref. [31]). 


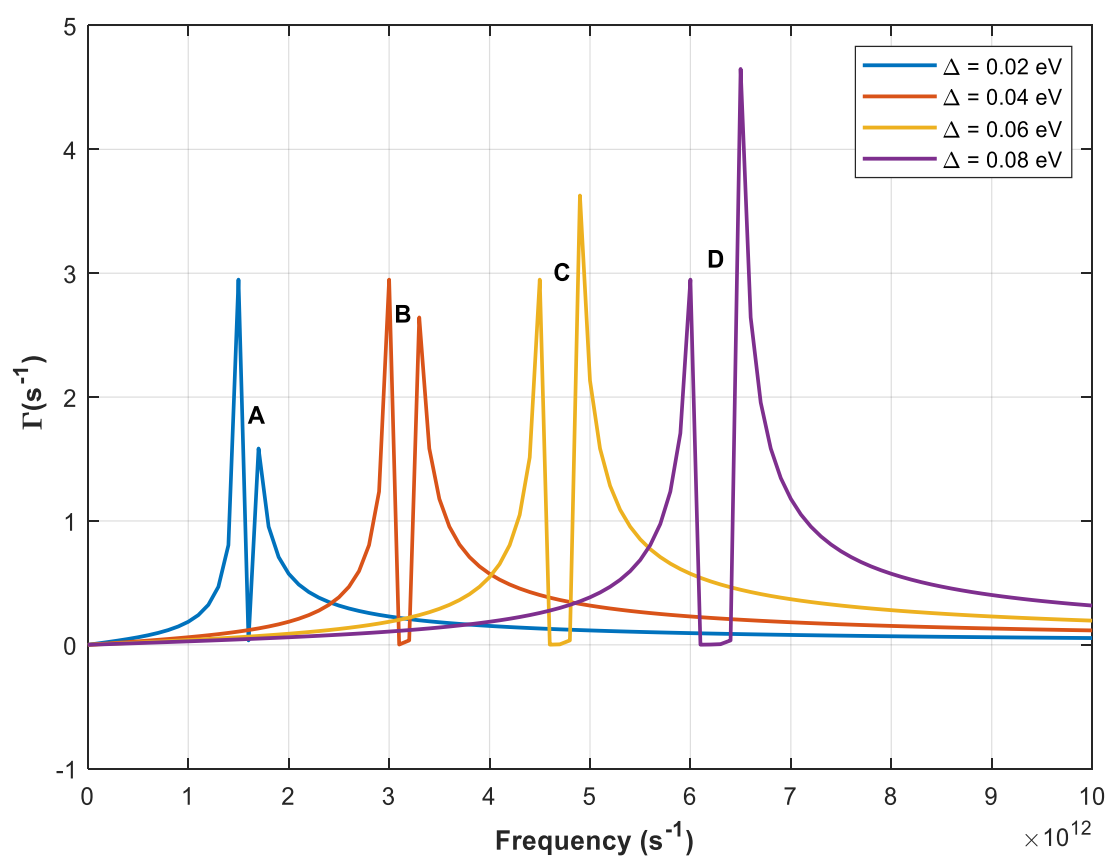

Fig 3: The dependence of $\Gamma_{q}$ on the $\omega_{q}$ showing absorption spectra with a broadening gap as the energy gap $(\Delta)$ value increases.

In Fig.3, we observed a twin peak with a varying peak height and gap between them that shifts to the right as the frequency increases. The first gap occurs at $\Delta=0.02 \mathrm{eV}$ whilst the second, third and fourth occur at 0.04, 0.06, $0.09 \mathrm{eV}$, respectively. From the plot, the gaps occur at points where the $\Gamma_{q}=0$. At A, we obtained a partial gap but B, C, and D showed a complete gap. When, $\Gamma_{q}=0$, from Eqn. (9), we obtain

$$
\Delta=\mathrm{h} \omega_{q}\left[1-\frac{v_{D}}{v_{s}}\right]
$$

Therefore, knowing $v_{D}, v_{s}$ and $\omega_{q}$, the energy gap $(\Delta)$ of the material can be determined as in Eqn. 10. From Eqn.(9), using the Weinrich relation, the acoustoelectric current was plotted against $\omega_{q}$ (see Fig. 4).The current increases to a maximum point (resonant) and then decreases. The plot is qualitatively consistent with experimental report by Bandu, et. al. [10], where the current obtained increases to a maximum point and then decreased. The maximum (resonant) point referred to as threshold frequency $\omega_{q}^{T H}$ beyond which the current decreases is dependent on the $v_{D}$. The plot shifts to the right when $v_{D}$ is increased making the $A E$ in graphene tunable. Unlike Fig.3 where for a certain quantized phonon energies, the absorption 
$\Gamma_{q}=0$, the absorption spectrum in Fig. $4 \mathrm{a}$ is not, due to inter band transitions where the conduction electrons in a bid to cross the energy gap loses their energy to the confined phonons. To further elucidation, a 3D plot of $J_{a c}$ versus $\omega_{q}$ and $q$ is displayed in Fig.4b. Similar results were obtained experimentally in the Megahertz (MHz) range by Liag, et. al [21], Okuda et. al [22], and Morgado et. al [23]. In the Terahertz range, simulated results of absorption in graphene obtained by Ullah, et. al. [26] is in qualitatively agreement with Fig. 4.
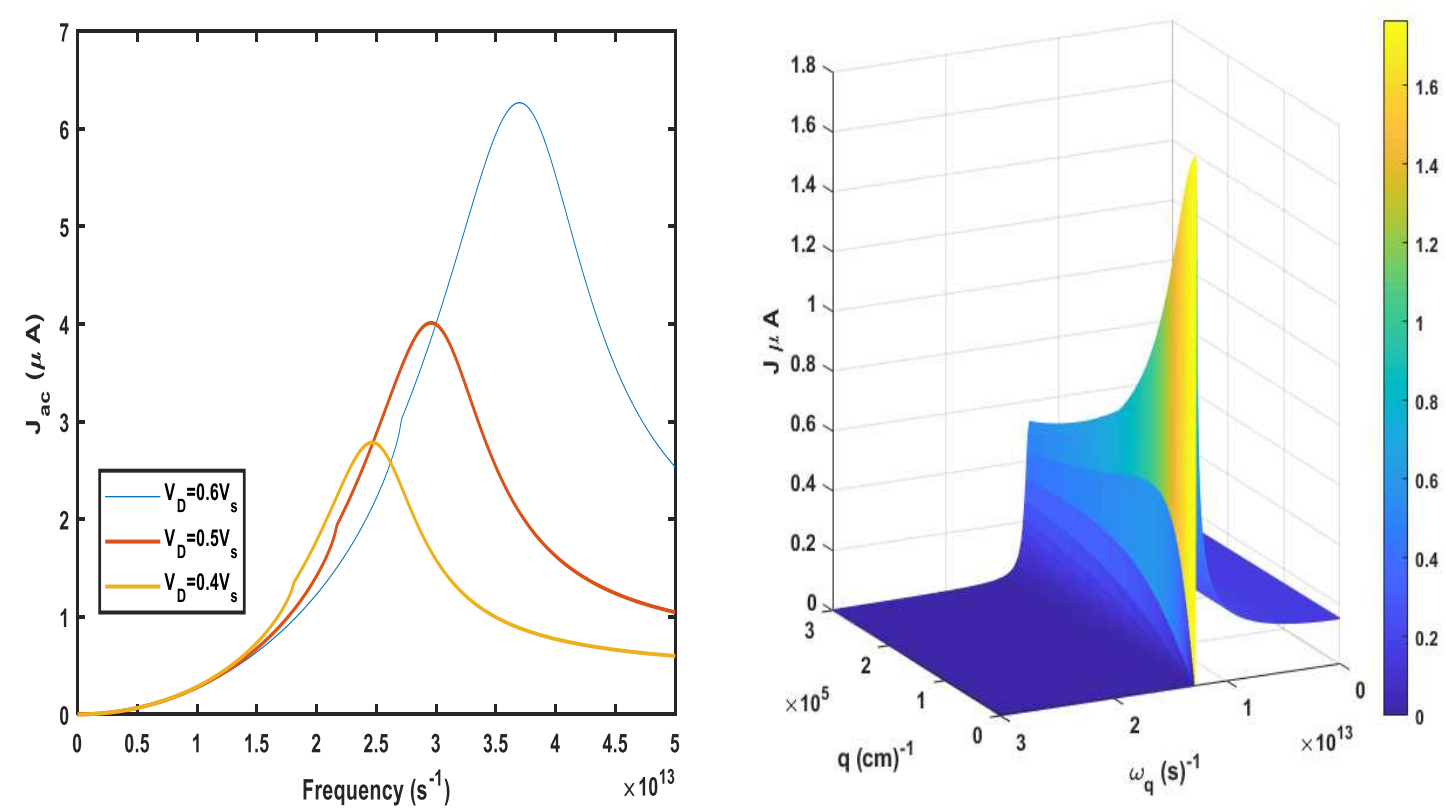

Fig 4: (a) Dependence of $J_{a c}$ on $\omega_{q}$ at various drift velocities $v_{D}$, and (b) a 3D plot of the dependence of $J_{a c}$ on $\omega_{q}$ and $q$.

\section{Conclusion}

We have demonstrated theoretically, the acoustoelectric current generation in GNR due to Landau damping of acoustic phonons when a drift electric field $\left(E_{D}\right)$ is applied. Other field such as temperature $(T)$ was applied to $S L G$ but the phenomenon of LDOAP was not observed. When a non-quantizing field was applied, the acoustoelectric current obtained shifts as the drift velocity is varied. This makes acoustoelectric current in GNR- tunable and a good acoustic wave filter for phonon spectroscopy. 


\section{Acknowledgments}

This work was partly supported by the Penn State-Altoona Office of Research and Sponsored Programs U.S.A, and the Directorate of research innovation and consultancy, University of Cape Coast, Ghana

\section{Reference}

[1] Landau, L. "On the vibration of the electronic plasma". JETP 16 (1946), 574. English translation in J. Phys. (USSR) 10 (1946), 25. Reproduced in Collected papers of L.D. Landau, edited and with an introduction by D. ter Haar, Pergamon Press, 1965, pp. 445-460; and in Men of Physics: L.D. Landau, Vol. 2, Pergamon Press, D. ter Haar, ed. (1965).

[2] G. Abstreiter, M. Cardona, and A. Pinczuk in: Light Scattering in Solids IV, edited by M. Cardona and M. Guntherödt, Topics in Applied Physics, Vol. 1 (Springer, Berlin, 1984), pp. $5-150$.

[3] G. R. Bell, C. F. McConville, and T. S. Jones, Phys. Rev. B 54, 2654 (1996).

[4] Mensah, S. Y., Allotey, F. K. A., \& Mensah, N. G. (2000). Nonlinear acoustoelectric effect in a semiconductor superlattice. Journal of Physics: Condensed Matter, 12(24), 5225.

[5] Mensah, S. Y., Allotey, F. K. A., Mensah, N. G., Akrobotu, H., \& Nkrumah, G. (2005). The influence of external electric field on acoustoelectric effect in a superlattice. Superlattices and Microstructures, 37(2), 87-97.

[6] Mensah, S. Y., Allotey, F. K. A., \& Adjepong, S. K. (1996). Acoustomagnetoelectric effect in a superlattice. Journal of Physics: Condensed Matter, 8(9), 1235.

[7] Dompreh, K. A., Mensah, S. Y., Abukari, S. S., Sam, F., \& Mensah, N. G. (2014). Amplification of Acoustic Waves in Graphene Nanoribbon in the Presence of External Electric and Magnetic Field. arXiv preprint arXiv:1410.8064.

[8] Poole, T., Bandhu, L., \& Nash, G. R. (2015). Acoustoelectric photoresponse in graphene. Applied Physics Letters, 106(13), 133107.

[9] Bandhu, L., Lawton, L. M., \& Nash, G. R. (2013). Macroscopic acoustoelectric charge transport in graphene. Applied Physics Letters, 103(13), 133101. 
[10] Poole, T., \& Nash, G. R. (2017). Acoustoelectric current in graphene nanoribbons. Scientific reports, 7(1), 1-9.

[11] VAN NGHIA, N. G. U. Y. E. N., HUONG, T. T. T., \& BAU, N. Q. (2010). The nonlinear acoustoelectric effect in a cylindrical quantum wire with an infinite potential. In Proc. Natl. Conf. Theor. Phys (Vol. 35, pp. 183-188).

[12] Dompreh, K. A., Mensah, N. G., Mensah, S. Y., Sam, F., \& Twum, A. K. (2015). Acoustoelectric Effect in degenerate Carbon Nanotube. arXiv preprint arXiv:1504.05484.

[13] Ebbecke, J., Strobl, C. J., \& Wixforth, A. (2004). Acoustoelectric current transport through single-walled carbon nanotubes. Physical Review B, 70(23), 233401.

[14] Reulet, B., Kasumov, A. Y., Kociak, M., Deblock, R., Khodos, I. I., Gorbatov, Y. B., ... \& Bouchiat, H. (2000). Acoustoelectric effects in carbon nanotubes. Physical Review Letters, 85(13), 2829.

[15] Bau, N. Q., \& Van Hieu, N. (2013). The quantum acoustoelectric current in a doped superlattice GaAs: Si/GaAs: Be. Superlattices and Microstructures, 63, 121-130.

[16] Dompreh, K. A., Mensah, N. G., \& Mensah, S. Y. (2015). Amplification of Hypersound in Graphene with degenerate energy dispersion. arXiv preprint arXiv:1503.07360.

[17] Nunes, O. A. C., \& Fonseca, A. L. A. (2012). Amplification of hippersound in graphene under external direct current electric field. Journal of Applied Physics, 112(4), 043707.

[18] Shinokita, K., Reimann, K., Woerner, M., Elsaesser, T., Hey, R., \& Flytzanis, C. (2016). Strong Amplification of Coherent Acoustic Phonons by Intraminiband Currents in a Semiconductor Superlattice. Physical review letters, 116(7), 075504.

[19] P. V. Santos, T. Schumann, M. H. Oliveira, J. M. J. Lopes, and H. Riechert, Appl. Phys. Lett. 102, 221907 (2013).

[20] E. F. Whitehead, E. M. Chick, L. Bandhu, L. M. Lawton, and G. R. Nash, Appl. Phys. Lett. 103, 063110 (2013)

[21] Liang, J., Yang, X., Zheng, S., Zhang, H., Zhang, D., Zhang, M., \& Pang, W. (2017, September). Manipulation of carriers in graphene using an on-chip acoustic wave device. In 2017 IEEE International Ultrasonics Symposium (IUS) (pp. 1-4). IEEE. 
[22] Okuda, S., Ikuta, T., Kanai, Y., Ono, T., Ogawa, S., Fujisawa, D., ... \& Matsumoto, K. (2016). Acoustic carrier transportation induced by surface acoustic waves in graphene in solution. Applied Physics Express, 9(4), 045104.

[23] Morgado, T. A., \& Silveirinha, M. G. (2017). Negative Landau damping in bilayer graphene. Physical review letters, 119(13), 133901.

[24] Mariani, E., \& Von Oppen, F. (2008). Flexural phonons in free-standing graphene. Physical review letters, 100(7), 076801.

[25] Weinreich G. 1956 Phys. Rev. 104321.

[26] Ullah, Z., Nawi, I., Witjaksono, G., Tansu, N., Khattak, M. I., Junaid, M., \& Magsi, S. A. (2020). Dynamic Absorption Enhancement and Equivalent Resonant Circuit Modeling of Tunable Graphene-Metal Hybrid Antenna. Sensors, 20(11), 3187.

[27] Zheng, S., Zhang, H., Feng, Z., Yu, Y., Zhang, R., Sun, C., \& Zhang, D. (2016). Acoustic charge transport induced by the surface acoustic wave in chemical doped graphene. Applied Physics Letters, 109(18), 183110.

[28] Sakyi-Arthur, D., Mensah, S. Y., Adu, K. W., Dompreh, K. A., Edziah, R., \& Mensah, N. G. (2020). Acoustoelectric Effect in Fluorinated Carbon Nanotube in the Absence of External Electric Field. World Journal of Condensed Matter Physics, 10(1), 1-11.

[29] Sakyi-Arthur, D., Mensah, S. Y., Mensah, N. G., Dompreh, K. A., \& Edziah, R. (2018). Absorption of Acoustic Phonons in Fluorinated Carbon Nanotube with Non-Parabolic, Double Periodic Band. Phonons in Low Dimensional Structures, InTech, London, 129-142.

[30] Sakyi-Arthur, D., Mensah, S. Y., Adu, K. W., Dompreh, K. A., Edziah, R., Mensah, N., \& Jebuni-Adanu, C. (2020). Semiconductor Fluorinated Carbon Nanotube as a Low Voltage Current Amplifier Acoustic Device. World Journal of Condensed Matter Physics, 10(01), 12.

[31] Wu, J. (2019). Tunable multi-band terahertz absorber based on graphene nano-ribbon metamaterial. Physics Letters A, 383(22), 2589-2593. 


\section{Supplementary Files}

This is a list of supplementary files associated with this preprint. Click to download.

- SupplementaryinformationNature.docx 